Ann. Biol. anim. Bioch. Biophys., 1979, 19 (3 B), 907-910.

\title{
Transit digestif chez le rat recevant de la caséine traitée en milieu alcalin
}

\author{
par B. POSSOMPES, Im Soon CHOI SCHIN, P. BESANÇON \\ Laboratoire de Physiologie de la Nutrition \\ Université de Montpellier II, 34060 Montpellier Cedex, France.
}

Summary. Digestive transit in rats receiving an alkali-treated casein.

Rats ingesting alkali-treated casein $\left(\mathrm{NaOH}, 0.2 \mathrm{~N}, 80^{\circ} \mathrm{C}, 1 \mathrm{hr}\right)$ showed diarrhea, a reduction of digestibility and pancreatic hypertrophy.

However, neither dry matter ingestion rhythms nor overall gastrointestinal transit time were modified. The diarrhea might account for poor digestive utilization of the protein.

\section{Introduction.}

Les protéines ayant subi un traitement alcalin relativement sévère sont toujours considérées comme étant sans toxicité (Nashef et al., 1977), malgré la formation de lysinoalanine ( $\varepsilon_{-}(\mathrm{DL}-2$-amino-2-carboxyethyl)-L-Lysine) dont la néphrotoxicité a été bien démontrée chez le rat (Woodard et Short, 1976) mais qui serait sans effet sur l'homme (De Groot et al., 1976).

Chez des rats recevant une caséine traitée en milieu alcalin nous avons observé des diarrhées, une diminution importante de la digestibilité des protéines ainsi qu'une hypertrophie pancréatique très nette. Afin de savoir si la baisse de digestibilité et les diarrhées sont dues à une accélération du transit nous avons réalisé une étude du temps de rétention gastro-intestinal global d'un marqueur ingéré lors d'un repas témoin.

\section{Matériel et méthodes.}

Protocole expérimental. - Les expériences sont réalisées sur des rats mâles de souche Sprague-Dawley pesant soit $70 \mathrm{à} 90 \mathrm{~g}$ soit $270 \mathrm{~g}$, soumis à une photopériode lumineuse de $12 \mathrm{~h}(8 \mathrm{~h}-20 \mathrm{~h})$. Ils sont nourris ad libitum el peuvent accéder à tout moment à la nourriture. Celle-ci est distribuée sous forme semi-liquide (40 p. 100 de matière sèche) et contient 10 p. 100 de protéines par rapport à la matière sèche, qui 
sont apportées soit par de la caséine normale (Merck), soit par de la caséine ayant subi un traitement alcalin (100 g de caséine dans un litre de solution de $\mathrm{NaOH} 0,2 \mathrm{~N}, 80^{\circ} \mathrm{C}$, $60 \mathrm{mn}$, neutralisée par $\mathrm{HCl}$ ef lyophilisée).

Après une période d'adaptation de 10 jours les rats reçoivent au cours d'un seul repas situé entre 20 et $22 \mathrm{~h}$ l'oxyde de chrome (au taux de $1 \mathrm{p}$. 100 de la matière sèche), dont on établit ensuite la cinétique d'excrétion. L'oxyde de chrome est en effet considéré comme un bon marqueur de la phase solide du bol alimentaire (Forman, Schneeman ef Weir, 1978). Les consommations alimentaires sont mesurées par période de $2 \mathrm{~h}$, les fèces sont récoltées également par période de $2 \mathrm{~h}$. Les expériences sont réalisées simultanément sur quatre rats et sont recommencées au moins deux fois.

Pour connaître l'effet d'une restriction alimentaire sur le transit un lot de rats de $200 \mathrm{~g}$ reçoit une quantité d'aliments égale à 50 p. 100 seulement de celle consommée par les rats nourris ad libitum.

Méthode de dosage ef expression des résultats.

L'oxyde de chrome est dosé suivant la méthode de Bolin, King et Klosterman, 1952.

Nous préférons exprimer le temps de rétention gastro-intestinal global par le temps nécessaire à l'apparition de la concentration maximale du marqueur dans les fèces. Nous avons également calculé le temps de rétention moyen ou coefficient $R$ de Castle (1956), le temps de transit moyen de Zierler (1958) ainsi que la période de décroissance de la concentration en marqueur dans les fèces (François, Compère ef Rondia, 1968). Toutefois ces paramètres n'ont qu'une valeur comparative ; en effet aucune précaution n'a été prise afin d'empêcher la coprophagie pour ne pas perturber le transit normal.

\section{Résultats.}

Consommations et rythmes alimentaires. - Les consommations alimentaires sont de l'ordre de $23 \mathrm{~g}$ de matière sèche par jour chez les jeunes rats et de $27 \mathrm{~g}$ chez les plus âgés ; la différence est statistiquement significative $(P<0,01)$. Avec la caséine traitée, les écarts en fonction de l'âge sont beaucoup plus marqués : les consommations sont respectivement de $9 \mathrm{~g}$ et de $26 \mathrm{~g}$.

Les rythmes alimentaires ne sont pas modifiés et l'ingestion est essentiellement nocturne. On observe également que les rythmes d'excrétion des fèces sont très marqués et que l'excrétion est plus importante la nuit.

Temps de rétention. - Les temps de rétention exprimés selon Castle ou Zierler ne présentent aucune différence significative en fonction de l'âge ou de la composition du régime ; ils varient de 19,8 à 23,5 h selon la première formulation ou de 16,6 à $18,7 \mathrm{~h}$ selon la seconde.

Chez les jeunes rats, quel que soit le régime, le délai d'apparition de la concentration maximale en marqueur est plus faible que chez les plus âgés : 10,5 $\pm 1 \mathrm{~h}$ avec la caséine normale et $9,5 \pm 2 \mathrm{~h}$ avec la caséine traitée au lieu de, respectivement, $11,5 \pm 1 \mathrm{~h}$ et $12 \pm 2 \mathrm{~h}$. Les périodes de décroissance des concentrations en marqueur sont par contre beaucoup plus longues chez les jeunes rats. Du fait de la grande varia- 
bilité des temps de rétention entre les rats ces valeurs indiquent des tendances plutôt que des différences bien marquées entre les jeunes et les rats plus gros (tabl. 1).

TABLEAU 1

Temps de rétention du marqueur en fonction de la taille des animaux ef de la nature des protéines ingérées

\begin{tabular}{|c|c|c|c|c|c|}
\hline $\begin{array}{l}\text { Poids } \\
\text { des rats } \\
\text { (g) }\end{array}$ & $\begin{array}{l}\text { Quantités } \\
\text { ingérées } \\
(\mathrm{g} \mathrm{MS} / \mathrm{j})\end{array}$ & $\begin{array}{c}\text { Temps nécessaire à } \\
\text { l'apparition du pic } \\
\text { de concentration } \\
\text { maximum de } \mathrm{Cr}_{2} \mathrm{O}_{3} \\
(\mathrm{en} \mathrm{h})\end{array}$ & $\begin{array}{l}\text { Temps de } \\
\text { (er } \\
\text { d'après } \\
\text { Zierler }\end{array}$ & $\begin{array}{l}\text { rétention } \\
\text { h) } \\
\text { d'après } \\
\text { Castle }\end{array}$ & $\begin{array}{l}\text { Période de } \\
\text { décroissance } \\
\text { de la concentration } \\
\text { en marqueur } \\
\text { (François) } \\
\text { (T } 1 / 2 \text { en } h)\end{array}$ \\
\hline \multicolumn{6}{|c|}{ Coséine normale } \\
\hline $\begin{array}{r}85 \\
270\end{array}$ & $\begin{array}{l}22,9 \pm 2,5(a) \\
27,0 \pm 2,6(a)\end{array}$ & $\begin{array}{l}10,5 \pm 1,0 \\
11,5 \pm 1,0(c)\end{array}$ & $\begin{array}{l}17,6 \pm 1,3 \\
17,7 \pm 2,1\end{array}$ & $\begin{array}{l}21,3 \pm 4,0 \\
19,9 \neq 1,6\end{array}$ & $\begin{array}{r}13,6 \\
9,1\end{array}$ \\
\hline \multicolumn{6}{|c|}{ Animaux restreints } \\
\hline 220 & 12,0 & $7,8 \pm 1,3(c, d)$ & $17,2 \pm 2,7$ & $21,2 \pm 4,6$ & 15,4 \\
\hline \multicolumn{6}{|c|}{ Caséine traitée } \\
\hline $\begin{array}{r}70 \\
270\end{array}$ & $\begin{aligned} 9,3 & \pm 2,1(\mathrm{a}, \mathrm{b}) \\
25,9 & \pm 2,7(\mathrm{~b})\end{aligned}$ & $\begin{array}{c}9,5 \pm 2,0 \\
12,0 \pm 1,8(d)\end{array}$ & $\begin{array}{l}16,6 \pm 3,1 \\
18,7 \pm 1,7\end{array}$ & $\begin{array}{l}19,8 \pm 4,8 \\
23,5 \pm 3,9\end{array}$ & $\begin{array}{l}15,4 \\
10,2\end{array}$ \\
\hline
\end{tabular}

Les valeurs accompagnées d'une même lettre sont statistiquement différentes $(P<0,01)$.

La restriction alimentaire, imposée à des rats de $220 \mathrm{~g}$ réduit le délai d'apparition de la concentration maximale $(7,8 \pm 1 \mathrm{~h})$ statistiquement plus court que chez les rats nourris ad libitum, et allonge la période de décroissance, par rapport aux rats de $270 \mathrm{~g}$. Ceci s'explique probablement par une coprophagie plus importante.

\section{Discussion ef conclusion.}

Il est évident que chez les rats de $270 \mathrm{~g}$, l'ingestion de caséine traitée ne provoque pas d'accélération du transit gastro-intestinal. Chez les rats de $70 \mathrm{~g}$, l'ingestion alimentaire est beaucoup plus faible ; ceci peut expliquer la diminution du délai d'apparition de la concentration maximale de marqueur dans les fèces. Les phénomènes de diarrhées observés lors de l'ingestion de caséine ayant subi un traitement alcalin ne peuvent donc être attribués qu'à une diminution de l'absorption consécutive, elle-même, à une aptitude plus faible de la protéine à être attaquée par les hydrolases.

Commission CNERNA Digestion-Absorption/Association des Physiologistes, Paris 5-6 octobre 1978.

Remerciements. - Ce travail a bénéficié de l'aide de la CNAMTS. 


\section{Références}

BOLIN D. W., KING R. P., KLOSTERMAN E. W., 1952. A simplified method for determination of chromic oxyde $\left(\mathrm{Cr}_{2} \mathrm{O}_{3}\right)$ when used as an index substance. Science, 116, 634-635.

CASTLE E. J., 1956. The rate of passage of foodstuffs through the alimentary tract of goat. I. - Studies on the adult animal fed on hay and concentrates. Brit. J. Nutr., 10, 15-23.

DE GROOT A. P., SLUMP P., FERON V. J., VAN BEEK L., 1976. Effects of alcali-treated preteins : feeding studies with free and protein-bound lysinoalanine in rats and other animals. J. Nutr., 106, 1527-1538.

FORMAN L. P., SCHNEEMAN B. O., WEIR W. C., 1978. Correlation of chromium sesquioxide and $\left({ }^{14} \mathrm{C}\right)$ cellulose as fecal markers in rats. Proc. Soc. exp. Biol. Med., 157, 418-420.

FRANÇOIS E., COMPĖRE R., RONDIA G., 1968. Etude comparée de la vitesse de passage des aliments et des résidus alimentaires non digérés dans le tractus digestif du rat et du mouton. Bull. Rech. agron. Gembloux, III, (4), 655-688.

NASHEF A. S., OSUGA D. T., LEE H. S., AHMED A. I., WHITAKER J. R., FEENEY R. E., 1977. Effect of alkali on proteins. Disulfides and their products. J. Agric. Food Chem., 25, 245-251.

WOODARD J. C., SHORT D. D., 1976. Renal toxicity of the NE-(DL-2-amino-2-carboxyethyl)-LLysine (lysinoalanine) in rats. Food cosmet. Toxicol. 15, 117-119.

ZIERLER K. L., 1958. A simplified explanation of the theory of indicator for measurement of fluid flow and volume and other distributive phenomena. Bull. Johns Hopkins Hosp., 103, 199-217. 